\title{
Periodic Solutions for $n$-Species Lotka-Volterra Competitive Systems with Pure Delays
}

\author{
Ahmadjan Muhammadhaji, Rouzimaimaiti Mahemuti, and Zhidong Teng \\ College of Mathematics and System Sciences, Xinjiang University, Urumqi 830046, China \\ Correspondence should be addressed to Ahmadjan Muhammadhaji; ahmatjanam@aliyun.com
}

Received 11 January 2015; Revised 22 March 2015; Accepted 2 April 2015

Academic Editor: Martin J. Bohner

Copyright (c) 2015 Ahmadjan Muhammadhaji et al. This is an open access article distributed under the Creative Commons Attribution License, which permits unrestricted use, distribution, and reproduction in any medium, provided the original work is properly cited.

\begin{abstract}
We study a class of periodic general $n$-species competitive Lotka-Volterra systems with pure delays. Based on the continuation theorem of the coincidence degree theory and Lyapunov functional, some new sufficient conditions on the existence and global attractivity of positive periodic solutions for the $n$-species competitive Lotka-Volterra systems are established. As an application, we also examine some special cases of the system, which have been studied extensively in the literature.
\end{abstract}

\section{Introduction}

As we know well, in recent years the application of the continuation theorem of the coincidence degree theory developed by Gaines and Mawhin to the existence of positive periodic solutions in population dynamical systems has been studied extensively [1-21]. For example, in [1-3, 12, 14-20], the authors studied existence of positive periodic solutions for population competition systems, in $[4,5,8,13,21]$, the authors studied existence of positive periodic solutions for population cooperative systems, in [9], the authors studied existence of positive periodic solutions for population predator-prey system, and in $[6,11]$, the authors studied existence of positive periodic solutions for single species systems. The continuation theorem is a powerful tool to study the existence of periodic solutions of periodic high-dimensional timedelayed problems. When dealing with time-delayed problem, it is very convenient and the result is relatively simple $[4,5]$.

Frequently, the environments of most natural populations undergo temporal variation, causing changes in the growth characteristics of these populations. One of the methods of incorporating temporal nonuniformity of the environments in models is to assume that the parameters are periodic with the same period of the time variable [14].

However, in the real world, the growth rate of a natural species will not often respond immediately to changes in its own population or that of an interacting species but will rather do so after a time lag [22]. Research [23,24] has shown that time delays have a great destabilizing influence on the species population. Usually, time delays are of two types: discrete delay and distributed time delay. For a competition system, competitors have both an instantaneous competition and a memory competition in the past. Therefore, we should introduce distributed delay into model foundation, which will have more resemblance to the real ecosystem. In fact, during the last decades, most of the authors study dynamics of population with delays [1-7, 10-21, 25-31], which is useful for the control of the population of mankind, animals, and environment.

It is well known that the focus in theoretical models of population and community dynamics must be not only on how populations depend on their own population densities or the population densities of other organisms but also on how populations change in response to the physical environment [19]. To consider periodic environmental factor, it is reasonable to study Lotka-Volterra systems with periodic coefficients. One of the celebrated population dynamical systems is Lotka-Volterra competition system. Since the Lotka-Volterra competition system has been established and was accepted by many scientists, now it has became the most important means to explain the ecological phenomenon. Recently, a great deal of Lotka-Volterra competition system 
with delays have been proposed to study the existence of periodic solutions $[1-3,12,14-21,25-31]$ and many good results were obtained by using of the continuation theorem [1-3, 12, 14-21].

In [1], the authors studied the following nonautonomous $N$-species Lotka-Volterra competitive systems with continuous time delays:

$$
\begin{aligned}
\dot{x}=x(t)\left[r_{i}(t)-\sum_{j=1}^{n} a_{i j}(t) x_{j}\left(t-\tau_{i j}(t)\right)\right] & \\
& i=1,2, \ldots, n .
\end{aligned}
$$

By using the method of coincidence degree and Lyapunov functional, a set of easily verifiable sufficient conditions of the existence and global attractivity of positive periodic solutions are established. In [2], the authors considered the following nonautonomous $N$-species Lotka-Volterra competitive systems with distributed time delays,

$$
\begin{aligned}
& \dot{u}=u(t)\left[r_{i}(t)-a_{i i}(t) u_{i}(t)\right. \\
&\left.-\sum_{j=1, j \neq i}^{n} a_{i j}(t) \int_{-T_{i j}}^{0} K_{i j}(s) u_{j}(t+s) d s\right], \\
& i=1,2, \ldots, n,
\end{aligned}
$$

and the periodic state dependent delay Lotka-Volterra competition system,

$$
\begin{array}{r}
\dot{u}=u(t)\left[r_{i}(t)-a_{i i}(t) u_{i}(t)\right. \\
\left.-\sum_{j=1, j \neq i}^{n} a_{i j}(t) u_{j}\left(t-\tau_{j}\left(t, u_{1}(t), \ldots, u_{n}(t)\right)\right)\right], \\
i=1,2, \ldots, n .
\end{array}
$$

By using the Mawhin's continuation theorem, the sufficient conditions on the existence of positive periodic solutions are established. In [3], the authors considered the following nonautonomous delay $N$-species competitive systems with delays:

$$
\begin{aligned}
\dot{u} & =u(t)\left[F_{i}\left(t, u_{i}\left(t-\tau_{i i}(t)\right)\right)\right. \\
& \left.-\sum_{j=1, j \neq i}^{n} a_{i j}(t) u_{j}\left(t-\tau_{i j}(t)\right)\right],
\end{aligned}
$$

By means of the Mawhin's continuation theorem and Lyapunov function method, the sufficient conditions for the existence and global attractivity of positive periodic solutions are established. In [21], the authors studied the following nonautonomous $N$-species Lotka-Volterra cooperative systems with continuous time delays and feedback controls:

$$
\begin{aligned}
& \dot{x}_{i}(t)=x_{i}(t)\left[r_{i}(t)-\sum_{l=1}^{m} a_{i i l}(t) x_{i}\left(t-\tau_{i i l}(t)\right)\right. \\
& \left.+\sum_{j \neq i}^{n} \sum_{l=1}^{m} a_{i j l}(t) x_{j}\left(t-\tau_{i j l}(t)\right)\right]-d_{i}(t) u_{i}(t)-e_{i}(t) \\
& \cdot u_{i}\left(t-\varepsilon_{i}(t)\right), \\
& \dot{u}_{i}(t)=-b_{i}(t) u_{i}(t)+\beta_{i}(t) x_{i}(t)+\gamma_{i}(t) x_{i}\left(t-\sigma_{i}(t)\right), \\
& i=1,2, \ldots, n .
\end{aligned}
$$

The sufficient conditions for the existence of positive periodic solutions are established, based on the Mawhin's continuation theorem.

Motivated by the above works, in this paper, we investigate the following $n$ species periodic Lotka-Volterra type competitive systems with pure delays

$$
\begin{aligned}
\dot{x}_{i}(t)=x_{i}(t)\left[r_{i}(t)-\sum_{l=1}^{m} a_{i i l}(t) x_{i}\left(t-\tau_{i i l}(t)\right)\right. & \\
\left.-\sum_{j \neq i}^{n} \sum_{l=1}^{m} a_{i i l}(t) \int_{-\tau_{i j l}}^{0} k_{i j l}(s) x_{j}(t+s) d s\right], & \\
i & =1,2, \ldots, n .
\end{aligned}
$$

By using the technique of coincidence degree developed by Gaines and Mawhin in [32], we will establish some new sufficient conditions, which guarantee that the system has at least one positive periodic solution. By means of the Lyapunov functionals we also will further establish the sufficient conditions on the global attractivity of the positive periodic solution.

\section{Preliminaries}

In system (6), we have that $x_{i}(t)(i=1,2, \ldots, n)$ represent the density of $n$ competitive species $x_{i}(i=1,2, \ldots, n)$ at time $t$, respectively; $r_{i}(t)(i=1,2, \ldots, n)$ represent the intrinsic growth rate of species $x_{i}(i=1,2, \ldots, n)$ at time $t$, respectively; $a_{i i l}(t)(i=1,2, \ldots, n, l=1,2, \ldots, m)$ represent the intrapatch restriction density of species $x_{i}(i=1,2, \ldots, n)$ at time $t$, respectively; $a_{i j l}(t)(l=1,2, \ldots, m, i \neq j, i, j=$ $1,2, \ldots, n)$ represent the competitive coefficients between $n$ species $x_{i}(i=1,2, \ldots, n)$ at time $t$, respectively. In this paper, we always assume the following.

(H1) $\tau_{i j l}>0$ and $\tau_{i i l}(t)(l=1,2, \ldots, m, i=1,2, \ldots, n)$, $r_{i}(t)(i=1,2, \ldots, n)$ are continuous $\omega$-periodic functions with $\tau_{i i l}^{\prime}(t)<1$ and $\int_{0}^{\omega} r_{i}(t) d t>0 . a_{i j l}(t)(i, j=1,2, l=$ $1,2, \ldots, m),(i=1,2, \ldots, n)$ are continuous positive $\omega$ periodic functions. $k_{i j l}(s)(i, j=1,2, \ldots, n ; l=1,2, \ldots, m)$ 
are nonnegative integrable functions on $\left[-\tau_{i j l}, 0\right]$ satisfying $\int_{-\tau_{i j l}}^{0} k_{i j l}(s) d s=1$.

From the viewpoint of mathematical biology, in this paper for system (6) we only consider the solution with the following initial conditions:

$$
x_{i}(t)=\phi_{i}(t), \quad \forall t \in[-\gamma, 0], i=1,2, \ldots, n,
$$

where $\phi_{i}(t)(i=1,2, \ldots, n)$ are nonnegative continuous functions defined on $[-\gamma, 0]$ satisfying $\phi_{i}(0)>0(i=$ $1,2, \ldots, n)$ with $\gamma=\max _{t \in[0, \omega]}\left\{\tau_{i j l}(t),(i, j=1,2, l=\right.$ $1,2, \ldots, m)\}$.

In this paper, for any $\omega$-periodic continuous function $f(t)$, we denote

$$
\begin{aligned}
f^{L} & =\min _{t \in[0, \omega]} f(t), \\
f^{M} & =\max _{t \in[0, \omega]} f(t), \\
\bar{f} & =\frac{1}{\omega} \int_{0}^{\omega} f(t) d t .
\end{aligned}
$$

In order to obtain the existence of positive $\omega$-periodic solutions of system (6), we will use the continuation theorem developed by Gaines and Mawhin in [32]. For the reader's convenience, we will introduce the continuation theorem in the following.

Let $X$ and $Z$ be two normed vector spaces. Let $L$ : Dom $L \subset X \rightarrow Z$ be a linear operator and $N: X \rightarrow Z$ a continuous operator. The operator $L$ is called a Fredholm operator of index zero, if $\operatorname{dim} \operatorname{Ker} L=$ co $\operatorname{dim} \operatorname{Im} L<\infty$ and $\operatorname{Im} L$ is a closed set in $Z$. If $L$ is a Fredholm operator of index zero, then there exist continuous projectors $P: X \rightarrow X$ and $Q: Z \rightarrow Z$ such that $\operatorname{Im} P=\operatorname{Ker} L$ and $\operatorname{Im} L=$ $\operatorname{Ker} Q=\operatorname{Im}(I-Q)$. It follows that $L \mid \operatorname{Dom} L \cap \operatorname{Ker} P$ : Dom $L \cap \operatorname{Ker} P \rightarrow \operatorname{Im} L$ is invertible and its inverse is denoted by $K_{P}$ and denote by $J: \operatorname{ImQ} \rightarrow \operatorname{Ker} L$ an isomorphism of $\operatorname{Im} Q$ onto $\operatorname{Ker} L$. Let $\Omega$ be a bounded open subset of $X$; we say that the operator $N$ is $L$-compact on $\bar{\Omega}$, where $\bar{\Omega}$ denotes the closure of $\Omega$ in $X$, if $Q N(\bar{\Omega})$ is bounded and $K_{P}(I-Q) N$ : $\bar{\Omega} \rightarrow X$ is compact. Such definitions can be found in $[4,5]$.

Lemma 1 (see [10]). Suppose $\tau \in C^{1}(R, R)$ with $\tau(t+\omega) \equiv \tau(t)$ and $\tau^{\prime}(t)<1, \forall t \in[0, \omega]$. Then the function $t-\tau(t)$ has a unique inverse function $\mu(t)$ satisfying $\mu \in C(R, R), \mu(u+\omega)=$ $\mu(u)+\omega, \forall u \in R$.

Lemma 2 (see [32]). Let $L$ be a Fredholm operator of index zero and let $N$ be L-compact on $\bar{\Omega}$. If

(a) for each $\lambda \in(0,1)$ and $x \in \partial \Omega \cap \operatorname{Dom} L, L x \neq \lambda N x$,

(b) for each $x \in \partial \Omega \cap \operatorname{Ker} L, Q N x \neq 0$,

(c) $\operatorname{deg}\{J Q N, \Omega \cap \operatorname{Ker} L, 0\} \neq 0$,

then the operator equation $L x=N x$ has at least one solution lying in $\operatorname{Dom} L \cap \bar{\Omega}$.

\section{Main Results}

Now, for convenience of statements we denote the functions

$$
a_{i j}(t)=\sum_{l=1}^{m} a_{i j l}(t), \quad i, j=1,2, \ldots, n .
$$

The following theorem is about the existence of positive periodic solutions of system (6).

Theorem 3. Suppose that assumption (H1) holds and there exists a constant $\theta_{i}>0, i=1,2, \ldots, n$, such that

$$
\min _{t \in \omega}\left\{\bar{r}_{i} \theta_{i}-\sum_{j \neq i}^{n} \sum_{l=1}^{m} a_{i j l}^{M} \theta_{j} \frac{\sum_{i=1}^{n} \bar{r}_{i}}{A_{j}}\right\}=: B_{i}>0,
$$

where

$$
\begin{aligned}
& \min _{t \in \omega}\left\{\sum_{l=1}^{m}\left(\delta_{i i l}(t)+\sum_{j \neq i}^{n} \int_{-\tau}^{0} a_{j i l}(t-s) k_{j i l}(s) d s\right)\right\} \\
& =: A_{i} \\
& \delta_{i i l}(t) \\
& =\frac{a_{i i l}\left(\varphi_{i i l}(t)\right)}{1-\tau_{i i l}^{\prime}\left(\varphi_{i i l}(t)\right)}, \quad i=1,2, \ldots, n, l=1,2, \ldots, m,
\end{aligned}
$$

and the algebraic equation,

$$
\bar{r}_{i}-\bar{a}_{i i} v_{i}-\sum_{j \neq i}^{n} \bar{a}_{i j} v_{j}=0, \quad i=1,2, \ldots, n,
$$

has a unique positive solution. Then system (6) has at least one positive $\omega$-periodic solution.

Proof. For system (6) we introduce new variables $y_{i}(t)(i=$ $1,2, \ldots, n)$ such that

$$
x_{i}(t)=\exp \left\{y_{i}(t)\right\}, \quad i=1,2, \ldots, n .
$$

Then system (6) is rewritten in the following form:

$$
\begin{array}{r}
\dot{y}_{i}(t)=r_{i}(t)-\sum_{l=1}^{m} a_{i i l}(t) \exp \left\{y_{i}\left(t-\tau_{i i l}(t)\right)\right\} \\
-\sum_{j \neq i}^{n} \sum_{l=1}^{m} a_{i j l}(t) \int_{-\tau_{i j l}}^{0} k_{i j l}(s) \exp \left\{y_{j}(t+s)\right\} d s, \\
i=1,2, \ldots, n .
\end{array}
$$

In order to apply Lemma 2 to system (14), we introduce the normed vector spaces $X$ and $Z$ as follows. Let $C\left(R, R^{n}\right)$ denote the space of all continuous function $y(t)=$ $\left(y_{1}(t), y_{2}(t), \ldots, y_{n}(t)\right): R \rightarrow R^{n}$. We take

$$
\begin{aligned}
X & =Z=\{y(t) \\
& \left.\in C\left(R, R^{n}\right): y(t) \text { is an } \omega \text {-periodic function }\right\},
\end{aligned}
$$


with norm

$$
\|y\|=\sum_{i=1}^{n} \max _{t \in[0, \omega]}\left|y_{i}(t)\right|
$$

It is obvious that $X$ and $Z$ are the Banach spaces. We define a linear operator $L:$ Dom $L \subset X \rightarrow Z$ and a continuous operator $N: X \rightarrow Z$ as follows:

$$
\begin{aligned}
L y(t) & =\dot{y}(t), \\
N y(t) & =\left(N y_{1}(t), N y_{2}(t), \ldots, N y_{n}(t)\right),
\end{aligned}
$$

where

$$
\begin{aligned}
N y_{i}(t) & \\
= & r_{i}(t)-\sum_{l=1}^{m} a_{i i l}(t) \exp \left\{y_{i}\left(t-\tau_{i i l}(t)\right)\right\} \\
& -\sum_{j \neq i}^{n} \sum_{l=1}^{m} a_{i j l}(t) \int_{-\tau_{i j l}}^{0} k_{i j l}(s) \exp \left\{y_{j}(t+s)\right\} d s .
\end{aligned}
$$

Further, we define continuous projectors $P: X \rightarrow X$ and $Q: Z \rightarrow Z$ as follows:

$$
\begin{aligned}
& P y(t)=\frac{1}{\omega} \int_{0}^{\omega} y(t) d t \\
& Q v(t)=\frac{1}{\omega} \int_{0}^{\omega} v(t) d t
\end{aligned}
$$

We easily see $\operatorname{Im} L=\left\{v \in Z: \int_{0}^{\omega} v(t) d t=0\right\}$ and $\operatorname{Ker} L=R^{n}$. It is obvious that $\operatorname{Im} L$ is closed in $Z$ and $\operatorname{dim} \operatorname{Ker} L=n$. Since for any $v \in Z$ there are unique $v_{1} \in R^{n}$ and $v_{2} \in \operatorname{Im} L$ with

$$
\begin{aligned}
v_{1} & =\frac{1}{\omega} \int_{0}^{\omega} v(t) d t, \\
v_{2}(t) & =v(t)-v_{1},
\end{aligned}
$$

such that $v(t)=v_{1}+v_{2}(t)$, we have co $\operatorname{dim} \operatorname{Im} L=n$. Therefore, $L$ is a Fredholm mapping of index zero. Furthermore, the generalized inverse (to $L) K_{p}: \operatorname{Im} L \rightarrow \operatorname{Ker} P \cap \operatorname{Dom} L$ is given in the following form:

$$
K_{p} v(t)=\int_{0}^{t} v(s) d s-\frac{1}{\omega} \int_{0}^{\omega} \int_{0}^{t} v(s) d s d t
$$

For convenience, we denote $F(t)=\left(F_{1}(t), F_{2}(t), \ldots, F_{n}(t)\right)$ as follows:

$$
\begin{aligned}
F_{i}(t)= & r_{i}(t)-\sum_{l=1}^{m} a_{i i l}(t) \exp \left\{y_{i}\left(t-\tau_{i i l}(t)\right)\right\} \\
& -\sum_{j \neq i}^{n} \sum_{l=1}^{m} a_{i j l}(t) \int_{-\tau_{i j l}}^{0} k_{i j l}(s) \exp \left\{y_{j}(t+s)\right\} d s .
\end{aligned}
$$

Thus, we have

$$
\begin{aligned}
Q N y(t)= & \frac{1}{\omega} \int_{0}^{\omega} F(t) d t \\
K_{p}(I-Q) N u(t)= & K_{p} I N u(t)-K_{p} Q N u(t) \\
= & \int_{0}^{t} F(s) d s-\frac{1}{\omega} \int_{0}^{\omega} \int_{0}^{t} F(s) d s d t \\
& +\left(\frac{1}{2}-\frac{t}{\omega}\right) \int_{0}^{\omega} F(s) d s .
\end{aligned}
$$

From formulas (23), we easily see that $Q N$ and $K_{p}(I-Q) N$ are continuous operators. Furthermore, it can be verified that $\overline{K_{p}(I-Q) N(\bar{\Omega})}$ is compact for any open bounded set $\Omega \subset$ $X$ by using Arzela-Ascoli theorem and $Q N(\bar{\Omega})$ is bounded. Therefore, $N$ is $L$-compact on $\bar{\Omega}$ for any open bounded subset $\Omega \subset X$.

Now, we reach the position to search for an appropriate open bounded subset $\Omega$ for the application of the continuation theorem (Lemma 2) to system (14).

Corresponding to the operator equation $L y(t)=\lambda N y(t)$ with parameter $\lambda \in(0,1)$, we have

$$
\dot{y}_{i}(t)=\lambda F_{i}(t), \quad i=1,2, \ldots, n,
$$

where $F_{i}(t)(i=1,2, \ldots, n)$ are given in $(22)$.

Assume that $y(t)=\left(y_{1}(t), y_{2}(t), \ldots, y_{n}(t)\right) \in X$ is a solution of system (24) for some parameter $\lambda \in(0,1)$. By integrating system $(24)$ over the interval $[0, \omega]$, we obtain

$$
\begin{aligned}
\int_{0}^{\omega} & {\left[r_{i}(t)-\sum_{l=1}^{m} a_{i i l}(t) \exp \left\{y_{i}\left(t-\tau_{i i l}(t)\right)\right\}\right.} \\
& \left.-\sum_{j \neq i}^{n} \sum_{l=1}^{m} a_{i j l}(t) \int_{-\tau_{i j l}}^{0} k_{i j l}(s) \exp \left\{y_{j}(t+s)\right\} d s\right] d t \\
& =0, \quad i=1,2, \ldots, n .
\end{aligned}
$$

Consequently,

$$
\begin{aligned}
& \int_{0}^{\omega}\left[\sum_{l=1}^{m} a_{i i l}(t) \exp \left\{y_{i}\left(t-\tau_{i i l}(t)\right)\right\}\right] d t \\
& \quad+\int_{0}^{\omega}\left[\sum_{j \neq i}^{n} \sum_{l=1}^{m} a_{i j l}(t) \int_{-\tau_{i j l}}^{0} k_{i j l}(s) \exp \left\{y_{j}(t+s)\right\} d s\right] d t \\
& \quad=\bar{r}_{i} \omega, \quad i=1,2, \ldots, n .
\end{aligned}
$$

Let $s_{i i l}(t)=t-\tau_{i i l}(t)(i=1,2, \ldots, n, l=1,2, \ldots, m)$; then from Lemma 1 and (H1) we get that function $s_{i i l}(t)$ has a unique $\omega$ periodic inverse function $\varphi_{i i l}(t)$; then for every $i=1,2, \ldots, n, l=1,2, \ldots, m$, we have

$$
\begin{aligned}
\int_{0}^{\omega} a_{i i l}(t) \exp \left\{y_{i}\left(t-\tau_{i i l}(t)\right)\right\} d t \\
\quad=\int_{-\tau_{i i l}(0)}^{\omega-\tau_{i i l}(\omega)} \frac{a_{i i l}\left(\varphi_{i i l}(t)\right)}{1-\tau_{i i l}^{\prime}\left(\varphi_{i i l}(t)\right)} \exp \left\{y_{i}(t)\right\} d t .
\end{aligned}
$$


One can see that

$$
\frac{a_{i i l}\left(\varphi_{i i l}(t)\right)}{1-\tau_{i i l}^{\prime}\left(\varphi_{i i l}(t)\right)}=: \delta_{i i l}(t)
$$

$$
i=1,2, \ldots, n, l=1,2, \ldots, m
$$

are $\omega$ periodic functions. Then for every $i=1,2, \ldots, n, l=$ $1,2, \ldots, m$, we have

$$
\begin{gathered}
\int_{0}^{\omega} a_{i i l}(t) \exp \left\{y_{i}\left(t-\tau_{i i l}(t)\right)\right\} d t \\
=\int_{0}^{\omega} \delta_{i i l}(t) \exp \left\{y_{i}(t)\right\} d t .
\end{gathered}
$$

For each $i, j=1,2, \ldots, n$, and $l=1,2, \ldots, m$, we have

$$
\begin{aligned}
\int_{0}^{\omega} & a_{i j l}(t) \int_{-\tau}^{0} k_{i j l}(s) \exp \left\{y_{j}(t+s)\right\} d s d t \\
& =\int_{-\tau}^{0} \int_{0}^{\omega} a_{i j l}(t) k_{i j l}(s) \exp \left\{y_{j}(t+s)\right\} d t d s \\
& =\int_{-\tau}^{0} \int_{s}^{s+\omega} a_{i j l}(v-s) k_{i j l}(s) \exp \left\{y_{j}(v)\right\} d v d s \\
& =\int_{-\tau}^{0} \int_{0}^{\omega} a_{i j l}(v-s) k_{i j l}(s) \exp \left\{y_{j}(v)\right\} d v d s \\
& =\int_{0}^{\omega} \int_{-\tau}^{0} a_{i j l}(v-s) k_{i j l}(s) \exp \left\{y_{j}(v)\right\} d s d v \\
& =\int_{0}^{\omega}\left(\int_{-\tau}^{0} a_{i j l}(t-s) k_{i j l}(s) d s\right) \exp \left\{y_{j}(t)\right\} d t .
\end{aligned}
$$

From $((26)-(30))$, we obtain

$$
\begin{aligned}
& \sum_{i=1}^{n}\left(\int_{0}^{\omega}\left[\sum_{l=1}^{m} \delta_{i i l}(t) \exp \left\{u_{i}(t)\right\}+\sum_{j \neq i}^{n} \sum_{l=1}^{m}\left(\int_{-\tau}^{0} a_{i j l}(t-s) k_{i j l}(s) d s\right) \exp \left\{u_{j}(t)\right\}\right] d t\right) \\
& \quad=\sum_{i=1}^{n}\left(\int_{0}^{\omega}\left[\sum_{l=1}^{m} \delta_{i i l}(t) \exp \left\{u_{i}(t)\right\}+\sum_{j \neq i}^{n} \sum_{l=1}^{m}\left(\int_{-\tau}^{0} a_{j i l}(t-s) k_{j i l}(s) d s\right) \exp \left\{u_{i}(t)\right\}\right] d t\right) \\
& \quad=\sum_{i=1}^{n}\left(\int_{0}^{\omega} \sum_{l=1}^{m}\left(\delta_{i i l}(t)+\int_{-\tau}^{0} \sum_{j \neq i}^{n} a_{j i l}(t-s) k_{j i l}(s) d s\right) \exp \left\{u_{i}(t)\right\} d t\right)=\sum_{i=1}^{n} \bar{r}_{i} \omega .
\end{aligned}
$$

From the above equality we have

$$
\begin{gathered}
\int_{0}^{\omega} \sum_{l=1}^{m}\left(\delta_{i i l}(t)+\int_{-\tau}^{0} \sum_{j \neq i}^{n} a_{j i l}(t-s) k_{j i l}(s) d s\right) \\
\cdot \exp \left\{u_{i}(t)\right\} d t \leq \sum_{i=1}^{n} \bar{r}_{i} \omega, \quad i=1,2, \ldots, n .
\end{gathered}
$$

From (32) we can obtain

$$
A_{i} \int_{0}^{\omega} \exp \left\{y_{i}(t)\right\} d t \leq \sum_{i=1}^{n} \bar{r}_{i} \omega, \quad i=1,2, \ldots, n,
$$

where

$A_{i}$

$$
=\min _{t \in \omega}\left\{\sum_{l=1}^{m}\left(\delta_{i i l}(t)+\sum_{j \neq i}^{n} \int_{-\tau}^{0} a_{j i l}(t-s) k_{j i l}(s) d s\right)\right\} .
$$

Consequently,

$$
\int_{0}^{\omega} \exp \left\{y_{i}(t)\right\} d t \leq \frac{\sum_{i=1}^{n} \bar{r}_{i} \omega}{A_{i}}, \quad i=1,2, \ldots, n .
$$

From the continuity of $y(t)=\left(y_{1}(t), y_{2}(t), \ldots, y_{n}(t)\right)$, there exist constants $\xi_{i}, \eta_{i} \in[0, \omega](i=1,2, \ldots, n)$ such that

$$
\begin{aligned}
y_{i}\left(\xi_{i}\right) & =\max _{t \in[0, \omega]} y_{i}(t), \\
y_{i}\left(\eta_{i}\right) & =\min _{t \in[0, \omega]} y_{i}(t), \\
i & =1,2, \ldots, n .
\end{aligned}
$$

From (35) and (36), we further obtain

$$
y_{i}\left(\eta_{i}\right) \leq \ln \left(\frac{\sum_{i=1}^{n} \bar{r}_{i}}{A_{i}}\right), \quad i=1,2, \ldots, n .
$$

From ((26)-(30)), we can obtain

$$
\begin{aligned}
& \int_{0}^{\omega}\left[\sum_{l=1}^{m} \delta_{i i l}(t) \exp \left\{y_{i}(t)\right\}\right] d t \\
& \quad+\sum_{j \neq i}^{n} \int_{0}^{\omega} \sum_{l=1}^{m} \int_{-\tau_{i j l}}^{0} a_{i j l}(t-s) k_{i j l}(s) d s \exp \left\{y_{j}(t)\right\} d t \\
& =\bar{r}_{i} \omega, \quad i=1,2, \ldots, n .
\end{aligned}
$$


From (38) we can obtain

$$
\begin{aligned}
& \sum_{l=1}^{m} \sigma_{i i l}^{M} \int_{0}^{\omega} \exp \left\{y_{i}(t)\right\} d t \\
& \quad \geq \bar{r}_{i} \omega-\sum_{j \neq i}^{n} \sum_{l=1}^{m} a_{i j l}^{M} \int_{0}^{\omega} \exp \left\{y_{j}(t)\right\} d t
\end{aligned}
$$

$$
i=1,2, \ldots, n \text {. }
$$

From the assumptions of Theorem 3 and (35), (38), and (39) we further obtain

$$
y_{i}\left(\xi_{i}\right) \geq \ln \left(\frac{B_{i}}{\sum_{l=1}^{m} \sigma_{i i l}^{M}}\right), \quad i=1,2, \ldots, n
$$

where

$$
B_{i}=\min _{t \in \omega}\left\{\bar{r}_{i}-\sum_{j \neq i}^{n} \sum_{l=1}^{m} a_{i j l}^{M} \frac{\sum_{i=1}^{n} \bar{r}_{i}}{A_{j}}\right\} .
$$

On the other hand, directly from system (14) we have

$$
\begin{aligned}
& \int_{0}^{\omega}\left|\dot{y}_{i}(t)\right| d t \leq \int_{0}^{\omega}\left|r_{i}(t)\right| d t \\
& +\int_{0}^{\omega}\left(\sum_{l=1}^{m} a_{i i l}(t) \exp \left\{y_{i}\left(t-\tau_{i i l}(t)\right)\right\}\right. \\
& \left.+\sum_{j \neq i}^{n} \sum_{l=1}^{m} a_{i j l}(t) \int_{-\tau_{i j l}}^{0} k_{i j l}(s) \exp \left\{y_{j}(t+s)\right\} d s\right) d t \\
& \quad \leq \int_{0}^{\omega}\left|r_{i}(t)\right| d t+\sum_{l=1}^{m} \delta_{i i l}^{M} \int_{0}^{\omega} \exp \left\{y_{i}(t)\right\} d t \\
& +\sum_{l=1}^{m} \sum_{j \neq i}^{n} a_{i j l}^{M} \int_{0}^{\omega} \exp \left\{y_{j}(t)\right\} d t \leq \int_{0}^{\omega}\left|r_{i}(t)\right| d t \\
& +\sum_{l=1}^{m} \delta_{i i l}^{M} \frac{\sum_{i=1}^{n} \bar{r}_{i} \omega}{A_{i}}+\sum_{l=1}^{m} \sum_{j \neq i}^{n} a_{i j l}^{M} \frac{\sum_{i=1}^{n} \bar{r}_{i} \omega}{A_{j}} \leq\left|\bar{r}_{i}\right| \omega \\
& +C_{i}, \quad i=1,2, \ldots, n,
\end{aligned}
$$

where

$$
\begin{aligned}
C_{i}=\sum_{l=1}^{m}\left(\delta_{i i l}^{M} \frac{\sum_{i=1}^{n} \bar{r}_{i} \omega}{A_{i}}+\sum_{j \neq i}^{n} a_{i j l}^{M} \frac{\sum_{i=1}^{n} \bar{r}_{i} \omega}{A_{j}}\right) & \\
& i=1,2, \ldots, n .
\end{aligned}
$$

From (37), (40), and (42), we have for any $t \in[0, \omega]$

$$
\begin{aligned}
& y_{i}(t) \leq y_{i}\left(\eta_{i}\right)+\int_{0}^{\omega}\left|\dot{y}_{i}(t)\right| d t \\
& \leq \ln \left(\frac{\sum_{i=1}^{n} \bar{r}_{i}}{A_{i}}\right)+\left|\bar{r}_{i}\right| \omega+C_{i}=: M_{i} \text {, } \\
& i=1,2, \ldots, n, \\
& y_{i}(t) \geq y_{i}\left(\xi_{i}\right)-\int_{0}^{\omega}\left|\dot{y}_{i}(t)\right| d t \\
& \geq \ln \left(\frac{B_{i}}{\sum_{l=1}^{m} \sigma_{i i l}^{M}}\right)-\left|\bar{r}_{i}\right| \omega-C_{i}=: N_{i}, \\
& i=1,2, \ldots, n \text {. }
\end{aligned}
$$

Therefore, from (44) and (45), we have

$$
\max _{t \in[0, \omega]}\left|y_{i}(t)\right| \leq \max \left\{\left|M_{i}\right|,\left|N_{i}\right|\right\}=: B_{i}
$$

$$
i=1,2, \ldots, n \text {. }
$$

It can be seen that the constants $B_{i}(i=1,2, \ldots, n)$ are independent of parameter $\lambda \in(0,1)$. For any $y=\left(y_{1}\right.$, $\left.y_{2}, \ldots, y_{n}\right) \in R^{n}$, from (18) we can obtain

$$
\begin{aligned}
& Q N y=\left(Q N y_{1}, Q N y_{2}, \ldots, Q N y_{n}\right), \\
& Q N y_{i}=\bar{r}_{i}-\bar{a}_{i i} \exp \left\{y_{i}\right\}-\sum_{j \neq i}^{n} \bar{a}_{i j} \exp \left\{y_{j}\right\}, \\
& i=1,2, \ldots, n .
\end{aligned}
$$

We consider the following algebraic equation:

$$
\bar{r}_{i}-\bar{a}_{i i} v_{i}-\sum_{j \neq i}^{n} \bar{a}_{i j} v_{j}=0 \quad i=1,2, \ldots, n .
$$

From the assumption of Theorem 3 , the equation has a unique positive solution $v^{*}=\left(v_{1}^{*}, v_{2}^{*}, \ldots, v_{n}^{*}\right)$. Hence, the equation QNy $=0$ has a unique solution $y^{*}=\left(y_{1}^{*}, y_{2}^{*}, \ldots, y_{n}^{*}\right)=$ $\left(\ln v_{1}^{*}, \ln v_{2}^{*}, \ldots, \ln v_{n}^{*}\right) \in R^{n}$.

Choosing constant $B>0$ large enough such that $\left|y_{1}^{*}\right|+$ $\left|y_{2}^{*}\right|+\cdots+\left|y_{n}^{*}\right|<B$ and $B>B_{1}+B_{2}+\cdots+B_{n}$, we define a bounded open set $\Omega \subset X$ as follows:

$$
\Omega=\{y \in X:\|y\|<B\} .
$$

It is clear that $\Omega$ satisfies conditions $(a)$ and $(b)$ of Lemma 2. On the other hand, by directly calculating we can obtain

$$
\begin{aligned}
& \operatorname{deg}\{J Q N, \Omega \cap \operatorname{Ker} L,(0,0, \ldots, 0)\} \\
& =\operatorname{sgn}\left|\begin{array}{cccc}
f_{y_{1}}^{1} & f_{y_{2}}^{1} & \cdots & f_{y_{n}}^{1} \\
f_{y_{1}}^{2} & f_{y_{2}}^{2} & \cdots & f_{y_{n}}^{2} \\
\ldots & \ldots & \ldots & \ldots \\
f_{y_{1}}^{n} & f_{y_{2}}^{n} & \ldots & f_{y_{n}}^{n}
\end{array}\right|,
\end{aligned}
$$


where

$$
\begin{array}{r}
f_{y_{j}}^{i}=-\bar{a}_{i j} \exp \left\{y_{j}^{*}\right\}, \quad i=j, \\
f_{y_{j}}^{i}=-\bar{a}_{i j} \exp \left\{y_{j}^{*}\right\}, \quad i \neq j, \\
i, j=1,2, \ldots, n .
\end{array}
$$

From the assumption of Theorem 3, we have

$$
\left|\begin{array}{cccc}
f_{y_{1}}^{1} & f_{y_{2}}^{1} & \cdots & f_{y_{n}}^{1} \\
f_{y_{1}}^{2} & f_{y_{2}}^{2} & \cdots & f_{y_{n}}^{2} \\
\cdots & \cdots & \cdots & \cdots \\
f_{y_{1}}^{n} & f_{y_{2}}^{n} & \cdots & f_{y_{n}}^{n}
\end{array}\right| \neq 0 .
$$

From this, we finally have

$$
\operatorname{deg}\{J Q N, \Omega \cap \operatorname{Ker} L,(0,0, \ldots, 0)\} \neq 0 .
$$

This shows that $\Omega$ satisfies condition (c) of Lemma 2 . Therefore, system (14) has a $\omega$-periodic solution $y^{*}(t)=$ $\left(y_{1}^{*}(t), y_{2}^{*}(t), \ldots, y_{n}^{*}(t)\right) \in \bar{\Omega}$. Finally, we have system (6) which has a positive $\omega$-periodic solution. This completes the proof.

Theorem 4. Suppose that the conditions of Theorem 3 hold and $\tau_{i i l}(t) \equiv 0(i, j=1,2, l=1,2, \ldots, m)$; further, there exists a constant $\pi_{i}>0(i=1,2, \ldots, n)$ such that

$$
\begin{aligned}
& \min _{t \in[0, \omega]}\left\{\sum_{l=1}^{m}\left(\pi_{i} a_{i i l}(t)-\sum_{j \neq i}^{n} \pi_{j} \int_{-\tau_{i j l}}^{0} a_{i j l}(t-s) k_{i j l}(s) d s\right)\right\} \\
& =: \delta_{i}>0, \quad i=1,2, \ldots, n
\end{aligned}
$$

then system (6) has a positive w-periodic solution which is globally attractive.

Proof. From Theorem 3, we can obtain that system (6) has a positive periodic solution.

Let $x^{*}(t)=\left(x_{1}^{*}(t), x_{2}^{*}(t), \ldots, x_{n}^{*}(t)\right)$ be a positive periodic solution of system (6). Choose positive constants $m_{i}>0$, $M_{i}>0$ such that

$$
m_{i} \leq x_{i}^{*}(t) \leq M_{i}, \quad i=1,2, \ldots, n .
$$

From the assumptions of Theorem 4 , there exist constant $\beta>$ 0 such that for all $t \geq 0$ we have

$$
\delta_{i} \geq \beta>0, \quad i=1,2, \ldots, n .
$$

Let $\left(x_{1}(t), x_{2}(t), \ldots, x_{n}(t)\right)$ be any solution of system (6); we define Lyapunov functional as follows:

$$
\begin{aligned}
& V_{i}(t)=\pi_{i}\left|\ln x_{i}^{*}(t)-\ln x_{i}(t)\right| \\
& \quad+\sum_{j \neq i}^{n} \sum_{l=1}^{m} \pi_{j} \int_{-\tau_{i j l}}^{0} k_{i j l}(s) \int_{t+s}^{t} a_{i j l}(\theta-s)\left|x_{j}^{*}(\theta)-x_{j}(\theta)\right| d \theta d s .
\end{aligned}
$$

Calculating the upper right derivation of $V_{i}(t)$ along system (6) for $i=1,2, \ldots, n$, we have

$$
\begin{aligned}
& D^{+} V_{i}(t)=\operatorname{sign}\left(x_{i}^{*}(t)-x_{i}(t)\right)\left[-\sum_{l=1}^{m} \pi_{i} a_{i i l}(t)\left(x_{i}^{*}(t)\right.\right. \\
& \left.\quad-x_{i}(t)\right)+\sum_{j \neq i}^{n} \sum_{l=1}^{m} \pi_{j} a_{i j l}(t) \\
& \left.\quad \int_{-\tau}^{0} k_{i j l}(s)\left(x_{j}^{*}(t+\theta)-x_{j}(t+\theta)\right) d s\right] \\
& +\sum_{j \neq i}^{n} \sum_{l=1}^{m} \pi_{j} \int_{-\tau}^{0} a_{i j l}(t-s) k_{i j l}(s) d s\left|x_{j}^{*}(t)-x_{j}(t)\right| \\
& \quad-\sum_{j \neq i}^{n} \sum_{l=1}^{m} \pi_{j} a_{i j l}(t) \\
& \quad \int_{-\tau}^{0} k_{i j l}(s)\left|x_{j}^{*}(t+\theta)-x_{j}(t+\theta)\right| d s \\
& \quad \leq-\sum_{l=1}^{m} \pi_{i} a_{i i l}(t)\left|x_{i}^{*}(t)-x_{i}(t)\right| \\
& +\sum_{j \neq i}^{n} \sum_{l=1}^{m} \pi_{j} \int_{-\tau}^{0} a_{i j l}(t-s) k_{i j l}(s) d s\left|x_{j}^{*}(t)-x_{j}(t)\right| .
\end{aligned}
$$

Further, we define a Lyapunov function as follows:

$$
V(t)=\sum_{i=1}^{n} V_{i}(t)
$$

Calculating the upper right derivation of $V(t)$, from (58) we finally can obtain, for all $t \geq 0$,

$$
D^{+} V(t) \leq-\sum_{i=1}^{n} \delta_{i}\left|x_{i}^{*}(t)-x_{i}(t)\right| .
$$

Integrating both sides of (60) from 0 to $t$ and by (56), we derive

$$
V(t)+\beta \int_{0}^{t}\left(\sum_{i=1}^{n}\left|x_{i}^{*}(s)-x_{i}(s)\right|\right) d s \leq V(0),
$$

$$
t \geq 0
$$

then

$$
\int_{0}^{t}\left(\sum_{i=1}^{n}\left|x_{i}^{*}(s)-x_{i}(s)\right|\right) d s \leq \frac{V(0)}{\beta}, \quad t \geq 0 .
$$

By the definition of $V(t)$ and (59) we have

$$
\sum_{i=1}^{n} \mu_{i}\left|\ln x_{i}^{*}(t)-\ln x_{i}(t)\right| \leq V(t) \leq V(0), \quad t \geq 0
$$


Therefore, for $i=1,2, \ldots, n$ we have

$$
\mu_{i}\left|\ln x_{i}^{*}(t)-\ln x_{i}(t)\right| \leq V(0), \quad t \geq 0,
$$

which, together with (55), lead to

$$
\begin{aligned}
m_{i} \exp \left\{\frac{-V(0)}{\mu_{i}}\right\} \leq x_{i}(t) \leq M_{i} \exp \left\{\frac{V(0)}{\mu_{i}}\right\} & \\
& i=1,2, \ldots, n
\end{aligned}
$$

and hence $\sum_{i=1}^{n}\left|x_{i}^{*}(t)-x_{i}(t)\right| \in L^{1}[0,+\infty)$. From the boundedness of $x_{i}^{*}(t)$ and (64), it follows that $x_{i}(t)(i=$ $1,2, \ldots, n)$ are bounded for $t \geq 0$. It is obvious that both $x_{i}(t)$ and $x_{i}^{*}(t)$ satisfy the equations of system (9); then by system (9) and the boundedness of $x_{i}(t)$ and $x_{i}^{*}(t)$ we know that the derivatives $\dot{x}_{i}(t)$ and $\dot{x}_{i}^{*}(t)$ are bounded. Furthermore, we can obtain that $\dot{x}_{i}^{*}(t)-\dot{x}_{i}(t)(i=1,2, \ldots, n)$ and their derivatives remain bounded on $[0,+\infty)$. Therefore $\sum_{i=1}^{n}\left|x_{i}^{*}(t)-x_{i}(t)\right|$ is uniformly continuous on $[0,+\infty)$. Thus from (62), we have

$$
\lim _{t \rightarrow+\infty} \sum_{i=1}^{n}\left|x_{i}^{*}(t)-x_{i}(t)\right|=0 .
$$

Therefore,

$$
\lim _{t \rightarrow+\infty}\left(x_{i}^{*}(t)-x_{i}(t)\right)=0, \quad i=1,2, \ldots, n .
$$

This completes the proof of Theorem 4 .

Corollary 5. Suppose that the conditions of Theorem 4 hold; then system (6) is permanent.

Proof. From the global attractivity of bounded positive solutions, we can get that the permanence of system (6).

\section{Applications}

In this section, to illustrate the generality of our result, we apply Theorem 3 to some particular Lotka-Volterra type competition systems with pure delays. Consider the following periodic $n$-species competition systems:

$$
\begin{aligned}
& \dot{x}_{i}(t)=x_{i}(t)\left[r_{i}(t)-\sum_{l=1}^{m} \sum_{j=1}^{n} a_{i j l}(t) x_{j}\left(t-\tau_{i j l}(t)\right)\right] \text {, } \\
& i=1,2, \ldots n \text {. } \\
& \dot{x}_{i}(t)=x_{i}(t) \\
& \cdot\left[r_{i}(t)-\sum_{j=1}^{n} \sum_{l=1}^{m} a_{i j l}(t) \int_{-\tau_{i j l}}^{0} k_{i j l}(s) x_{j}(t+s) d s\right], \\
& i=1,2, \ldots n \text {. } \\
& \dot{x}_{i}(t)=x_{i}(t)\left[r_{i}(t)-\sum_{l=1}^{m} \sum_{j=1}^{n} a_{i j l}(t) x_{j}\left(t-\tau_{i j l}\right)\right] \text {, } \\
& i=1,2, \ldots, n \text {. }
\end{aligned}
$$

For system $((68)-(70))$, we assume the following:

(H2) $\tau_{i j l}(t)(l=1,2, \ldots, m, i, j=1,2, \ldots, n), r_{i}(t)(i=$ $1,2, \ldots, n)$ are continuous $\omega$-periodic functions with $\tau_{i j l}^{\prime}(t)<$ 1 and $\int_{0}^{\omega} r_{i}(t) d t>0 . a_{i j l}(t)(i, j=1,2, l=1,2, \ldots, m)$ are continuous, positive $\omega$-periodic functions.

(H3) $r_{i}(t)(i=1,2, \ldots, n)$ are $\omega$-periodic continuous functions with $\int_{0}^{\omega} r_{i}(t) d t>0 ; a_{i j l}(t)(i, j=1,2, \ldots, n ; l=$ $1,2, \ldots, m)$ are positive $\omega$-periodic continuous functions; $k_{i j l}(s)(i, j=1,2, \ldots, n ; l=1,2, \ldots, m)$ are nonnegative integrable functions on $\left[-\tau_{i j l}, 0\right](i, j=1,2, \ldots, n ; l=$ $1,2, \ldots, m)$ satisfying $\int_{-\tau_{i j l}}^{0} k_{i j l}(s) d s=1$.

(H4) $\tau_{i j l}>0(l=1,2, \ldots, m, i, j=1,2, \ldots, n)$ are constants, $r_{i}(t)(i=1,2, \ldots, n)$ are continuous $\omega$-periodic functions with $\int_{0}^{\omega} r_{i}(t) d t>0 . a_{i j l}(t)(i, j=1,2, l=$ $1,2, \ldots, m)$ are continuous, positive $\omega$-periodic functions. Thus from Theorem 3 we have the following.

Corollary 6. Suppose that assumption (H2) holds and there exists a constant $\zeta_{i}>0, i=1,2, \ldots, n$, such that

$$
\begin{array}{r}
\min _{t \in[0, \omega]}\left\{\left[\bar{r}_{i} \zeta_{i}-\sum_{j \neq i}^{n} \sum_{l=1}^{m} \delta_{i j l}^{M} \zeta_{j} \frac{\sum_{i=1}^{n} \bar{r}_{i}}{E_{j}}\right]\right\}= \\
=D_{i}>0, \\
i=1,2, \ldots, n,
\end{array}
$$

where

$$
\begin{aligned}
& \min _{t \in[0, \omega]}\left\{\sum_{l=1}^{m}\left[\delta_{i i l}(t)+\sum_{j \neq i}^{n} \delta_{j i l}(t)\right]\right\} \\
& =: E_{i}, \quad i=1,2, \ldots, n, \\
& \delta_{i j l}(t) \\
& =\frac{a_{i j l}\left(\varphi_{i j l}(t)\right)}{1-\tau_{i j l}^{\prime}\left(\varphi_{i j l}(t)\right)}, \\
& \quad i, j=1,2, \ldots, n, l=1,2, \ldots, m,
\end{aligned}
$$

and the algebraic equation,

$$
\bar{r}_{i}-\bar{a}_{i i} v_{i}-\sum_{j \neq i}^{n} \bar{a}_{i j} v_{j}=0, \quad i=1,2, \ldots, n,
$$

has a unique positive solution. Then system (68) has at least one positive $\omega$-periodic solution.

Corollary 7. Suppose that assumption (H3) holds and there exists a constant $\beta_{i}>0, i=1,2, \ldots, n$, such that

$$
\begin{array}{r}
\min _{t \in[0, \omega]}\left\{\left[\bar{r}_{i} \beta_{i}-\sum_{j \neq i}^{n} \sum_{l=1}^{m} a_{i j l}^{M} \beta_{j} \frac{\sum_{i=1}^{n} \bar{r}_{i}}{K_{j}}\right]\right\}= \\
=G_{i}>0, \\
i=1,2, \ldots, n
\end{array}
$$


where

$$
\begin{aligned}
& \min _{t \in[0, \omega]}\left\{\sum_{l=1}^{m} \int_{-\tau}^{0}\left[a_{i i l}(t-s) k_{i i l}(s)+\sum_{j \neq i}^{n} a_{j i l}(t-s) k_{j i l}(s)\right] d s\right\} \\
& =: K_{i}, \quad i=1,2, \ldots, n,
\end{aligned}
$$

and the algebraic equation,

$$
\bar{r}_{i}-\bar{a}_{i i} v_{i}-\sum_{j \neq i}^{n} \bar{a}_{i j} v_{j}=0, \quad i=1,2, \ldots, n,
$$

has a unique positive solution. Then system (69) has at least one positive $\omega$-periodic solution.

Corollary 8. Suppose that assumption (H4) holds and there exists a constant $\gamma_{i}>0, i=1,2, \ldots, n$, such that

$$
\begin{array}{r}
\min _{t \in[0, \omega]}\left\{\left[\bar{r}_{i} \gamma_{i}-\sum_{j \neq i}^{n} \sum_{l=1}^{m} a_{i j l}^{M} \gamma_{j} \frac{\sum_{i=1}^{n} \bar{r}_{i}}{L_{j}}\right]\right\}= \\
=H_{i}>0, \\
i=1,2, \ldots, n
\end{array}
$$

where

$$
\begin{array}{r}
\min _{t \in[0, \omega]}\left\{\sum_{l=1}^{m}\left[a_{i i l}\left(t+\tau_{i i l}\right)+\sum_{j \neq i}^{n} a_{j i l}\left(t+\tau_{j i l}\right)\right]\right\}=: L_{i}, \\
i=1,2, \ldots, n,
\end{array}
$$

and the algebraic equation,

$$
\bar{r}_{i}-\bar{a}_{i i} v_{i}-\sum_{j \neq i}^{n} \bar{a}_{i j} v_{j}=0, \quad i=1,2, \ldots, n,
$$

has a unique positive solution. Then system (70) has at least one positive $\omega$-periodic solution.

\section{One Example}

In this section, we will give one example to illustrate the results obtained in this paper. From the example, we will see that if the conditions of Theorem 3 hold, then the system has a positive periodic solution. If the conditions of Theorem 4 hold, then the system has a positive periodic solution and which is globally attractive.
Example. We consider the following periodic three-species competition systems:

$$
\begin{aligned}
\dot{x}_{1}(t) & =x_{1}(t)[1.1+\cos (t) \\
- & (6+\sin (t)) x_{1}\left(t-\tau_{111}(t)\right) \\
- & \left(\frac{22+11 \sin (t)}{20}\right) \int_{-\tau_{121}}^{0} k_{121}(s) x_{2}(t+s) d s \\
- & \left.\left(\frac{22+11 \sin (t)}{20}\right) \int_{-\tau_{131}}^{0} k_{131}(s) x_{3}(t+s) d s\right], \\
\dot{x}_{2} & (t)=x_{2}(t)[1.1+\sin (t) \\
- & (6+\sin (t)) x_{2}\left(t-\tau_{221}(t)\right) \\
- & \left(\frac{22+11 \sin (t)}{20}\right) \int_{-\tau_{211}}^{0} k_{211}(s) x_{1}(t+s) d s \\
- & \left.\left(\frac{2+\sin (t)}{2}\right) \int_{-\tau_{231}}^{0} k_{231}(s) x_{3}(t+s) d s\right] \\
\dot{x}_{3}(t) & =x_{3}(t)[1.1+\cos (t) \\
& -(t)) x_{3}\left(t-\tau_{331}(t)\right) \\
& (6+\cos (t) \\
20 & k_{311}(s) x_{1}(t+s) d s \\
- &
\end{aligned}
$$

where $\tau_{111}(t)=\tau_{221}(t)=\tau_{331}(t)=0, n=3, m=1, \omega=2 \pi$; by direct calculation we can get

$$
\begin{aligned}
& B_{1}=0.5, \\
& B_{2}=0.65, \\
& B_{3}=1.25,
\end{aligned}
$$

and the following system of equations has a unique positive solution

$$
\begin{gathered}
2 v_{1}-0.5 v_{2}-0.5 v_{3}=1.9802, \\
2 v_{2}-0.5 v_{1}-0.4 v_{3}=1.9608 \\
3 v_{3}-0.75 v_{1}-0.5 v_{2}=1.9417
\end{gathered}
$$

and get

$$
\begin{aligned}
& v_{1}=1.7554, \\
& v_{2}=1.6929, \\
& v_{3}=1.3682 .
\end{aligned}
$$

It is clear that all the conditions of Theorems 3 and 4 hold.

From the Figure 1. we can see, system (80) is permanent and has a positive periodic solution which is globally attractive. 


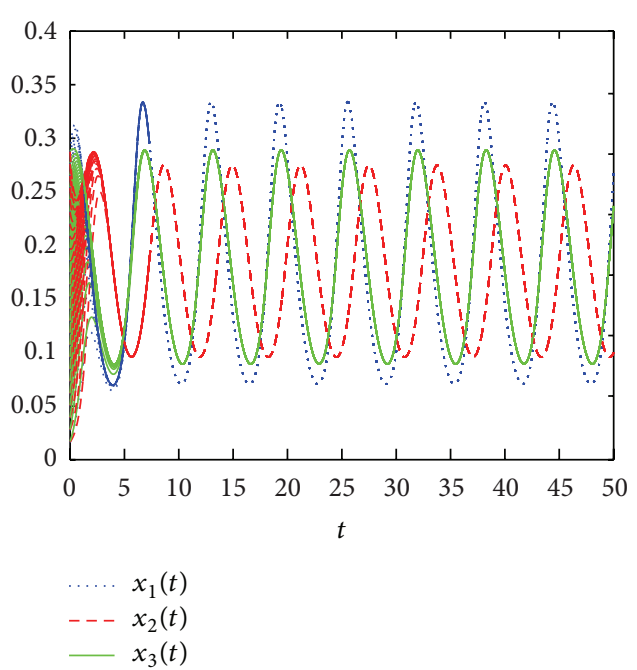

(a)

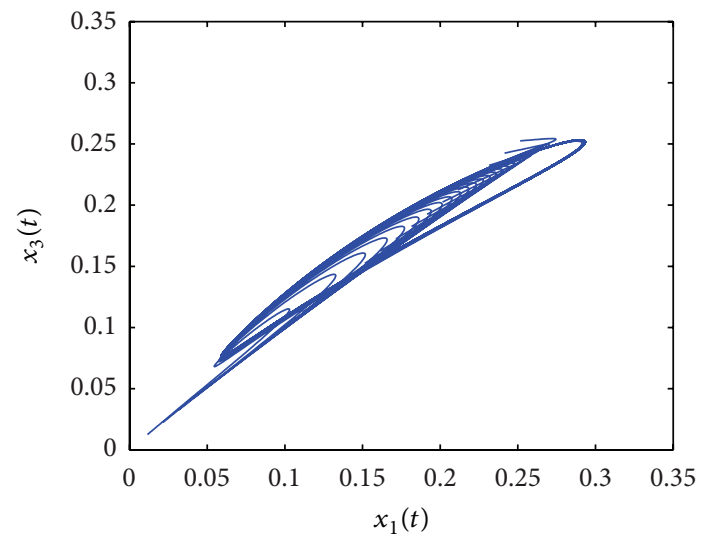

(c)

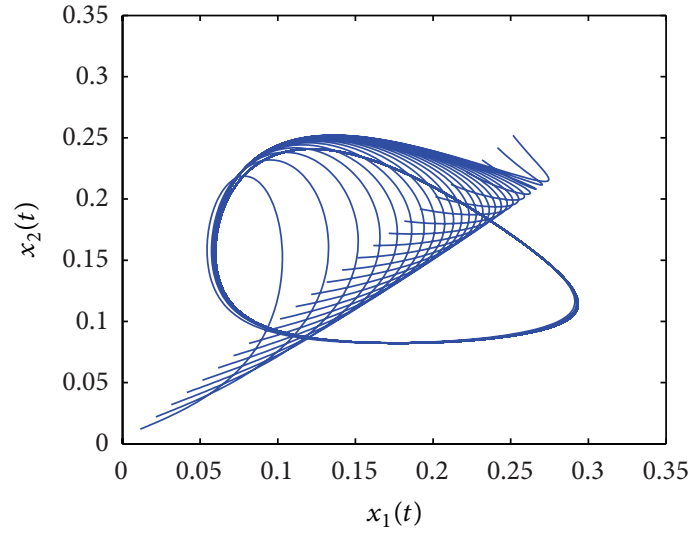

(b)

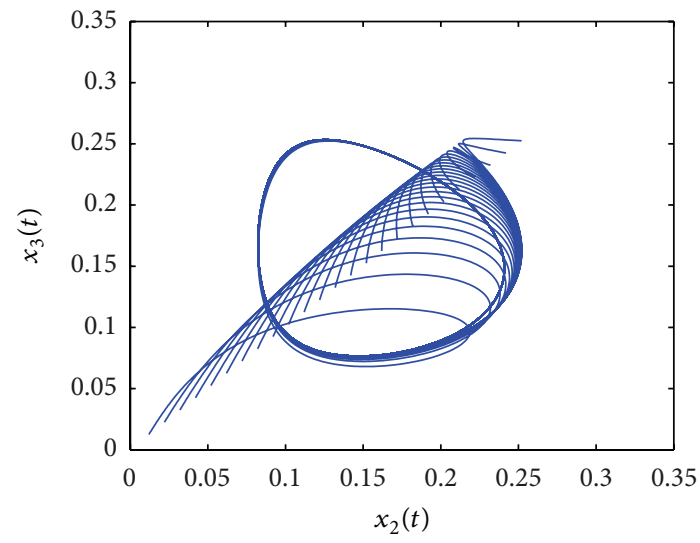

(d)

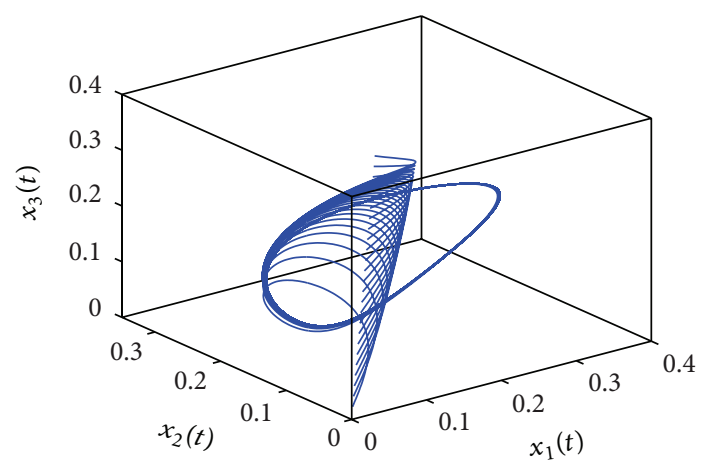

(e)

FIgURE 1: Dynamic behaviors of system (80).

\section{Conflict of Interests}

The authors declare that there is no conflict of interests regarding the publication of this paper.

\section{Acknowledgments}

This work was supported by the National Natural Science Foundation of China (Grants nos. 11271312, 11261056,
11261058, and 11401509) and the Natural Science Foundation of Xinjiang University (Starting Fund for Doctors, Grant no. Z01724).

\section{References}

[1] M. Fan, K. Wang, and D. Jiang, "Existence and global attractivity of positive periodic solutions of periodic $n$-species 
Lotka-Volterra competition systems with several deviating arguments," Mathematical Biosciences, vol. 160, no. 1, pp. 47-61, 1999.

[2] Y. Li, "Periodic solutions for delay Lotka-Volterra competition systems," Journal of Mathematical Analysis and Applications, vol. 246, no. 1, pp. 230-244, 2000.

[3] C. Egami, "Positive periodic solutions of nonautonomous delay competitive systems with weak Allee effect," Nonlinear Analysis: Real World Applications, vol. 10, no. 1, pp. 494-505, 2009.

[4] A. Muhammadhaji and Z. Teng, "Positive periodic solutions of $n$-species Lotka-Volterra cooperative systems with delays," Vietnam Journal of Mathematics, vol. 40, no. 4, pp. 453-467, 2012.

[5] A. Muhammadhaji and Z. Teng, "Global attractivity of a periodic delayed $N$-species model of facultative mutualism," Discrete Dynamics in Nature and Society, vol. 2013, Article ID 580185, 11 pages, 2013.

[6] H.-F. Huo and W.-T. Li, "Positive periodic solutions of a class of delay differential system with feedback control," Applied Mathematics and Computation, vol. 148, no. 1, pp. 35-46, 2004.

[7] F. Chen, "Positive periodic solutions of neutral Lotka-Volterra system with feedback control," Applied Mathematics and Computation, vol. 162, no. 3, pp. 1279-1302, 2005.

[8] D. Hu and Z. Zhang, "Four positive periodic solutions to a Lotka-Volterra cooperative system with harvesting terms," Nonlinear Analysis: Real World Applications, vol. 11, no. 2, pp. 1115-1121, 2010.

[9] K. Zhao and Y. Ye, "Four positive periodic solutions to a periodic Lotka-Volterra predatory-prey system with harvesting terms," Nonlinear Analysis. Real World Applications, vol. 11, no. 4, pp. 2448-2455, 2010.

[10] S. Lu, "On the existence of positive periodic solutions to a Lotka Volterra cooperative population model with multiple delays," Nonlinear Analysis: Theory, Methods \& Applications, vol. 68, no. 6, pp. 1746-1753, 2008.

[11] F. Yin and Y. Li, "Positive periodic solutions of a single-species model with feedback regulation and distributed time delay," Applied Mathematics and Computation, vol. 153, no. 2, pp. 475484, 2004.

[12] M. Kouche, N. Tatar, and S. Liu, "Permanence and existence of a positive periodic solution to a periodic stage-structured system with infinite delay," Applied Mathematics and Computation, vol. 202, no. 2, pp. 620-638, 2008.

[13] Z. Zhang, J. Wu, and Z. Wang, "Periodic solutions of nonautonomous stage-structured cooperative system," Computers \& Mathematics with Applications, vol. 47, no. 4-5, pp. 699-706, 2004.

[14] L. Zhang, H.-X. Li, and X.-B. Zhang, "Periodic solutions of competition Lotka-Volterra dynamic system on time scales," Computers \& Mathematics with Applications, vol. 57, no. 7, pp. 1204-1211, 2009.

[15] H. Fang and Y. Xiao, "Existence of multiple periodic solutions for delay Lotka-Volterra competition patch systems with harvesting," Applied Mathematical Modelling, vol. 33, no. 2, pp. 1086-1096, 2009.

[16] H. Fang and Z. Wang, "Existence and global attractivity of positive periodic solutions for delay Lotka-Volterra competition patch systems with stocking," Journal of Mathematical Analysis and Applications, vol. 293, no. 1, pp. 190-209, 2004.

[17] P. Weng, "Existence and global stability of positive periodic solution of periodic integrodifferential systems with feedback controls," Computers \& Mathematics with Applications, vol. 40, no. 6-7, pp. 747-759, 2000.

[18] M. Fan and K. Wang, "Global periodic solutions of a generalized $n$-species Gilpin-Ayala competition model," Computers \& Mathematics with Applications, vol. 40, no. 10-11, pp. 1141-1151, 2000.

[19] Y. Li, K. Zhao, and Y. Ye, "Multiple positive periodic solutions of $n$ species delay competition systems with harvesting terms," Nonlinear Analysis: Real World Applications, vol. 12, no. 2, pp. 1013-1022, 2011.

[20] C. $\mathrm{Xu}$ and $\mathrm{Y} . \mathrm{Wu}$, "Positive periodic solutions in a discrete time three species competition system," Journal of Applied Mathematics, vol. 20113, Article ID 963046, 13 pages, 2013.

[21] T. Niyaz and A. Muhammadhaji, "Positive periodic solutions of cooperative systems with delays and feedback controls," International Journal of Differential Equations, vol. 2013, Article ID 502963, 9 pages, 2013.

[22] A. Muhammadhaji, Z. Teng, and L. Zhang, "Permanence in general non-autonomous Lotka-Volterra predator-prey systems with distributed delays and impulses," Journal of Biological Systems, vol. 21, no. 2, Article ID 1350012, 28 pages, 2013.

[23] M. S. Bartlett, Stochastic Population Models, Methuen Publishing, London, UK, 1960.

[24] R. M. May, "Time-delay versus stability in population models with two and three trophic levels," Ecology, vol. 54, no. 2, pp. 315-325, 1973.

[25] X. Lv, P. Yan, and S. Lu, "Existence and global attractivity of positive periodic solutions of competitor-competitor-mutualist Lotka-Volterra systems with deviating arguments," Mathematical and Computer Modelling, vol. 51, no. 5-6, pp. 823-832, 2010.

[26] S. Tang, Y. Xiao, and J. Chen, "Positive periodic solutions of competitive Kolmogorov diffusion systems with interference constants," Mathematical and Computer Modelling, vol. 27, no. 6, pp. 39-47, 1998.

[27] Y. Chen and Z. Zhou, "Stable periodic solution of a discrete periodic Lotka-Volterra competition system," Journal of Mathematical Analysis and Applications, vol. 277, no. 1, pp. 358-366, 2003.

[28] X. Tang and X. Zou, "On positive periodic solutions of LotkaVolterra competition systems with deviating arguments," Proceedings of the American Mathematical Society, vol. 134, no. 10, pp. 2967-2974, 2006.

[29] F. Chen, "On the periodic solutions of periodic multi-species Kolmogorov type competitive system with delays and feedback controls," Applied Mathematics and Computation, vol. 180, no. 1, pp. 366-373, 2006.

[30] J. Yan, "Global positive periodic solutions of periodic $n$-species competition systems," Journal of Mathematical Analysis and Applications, vol. 356, no. 1, pp. 288-294, 2009.

[31] J. Yan and G. Liu, "Periodicity and stability for a LotkaVolterra type competition system with feedback controls and deviating arguments," Nonlinear Analysis: Theory, Methods \& Applications, vol. 74, no. 9, pp. 2916-2928, 2011.

[32] R. E. Gaines and J. L. Mawhin, Coincidence Degree and Nonlinear Differential Equations, vol. 568 of Lecture Notes in Mathematics, Springer, Berlin, Germany, 1977. 


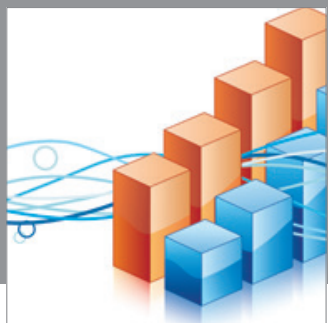

Advances in

Operations Research

mansans

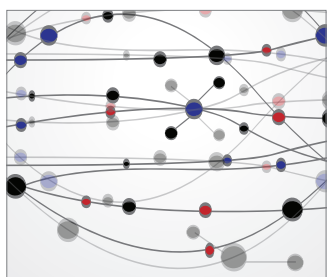

The Scientific World Journal
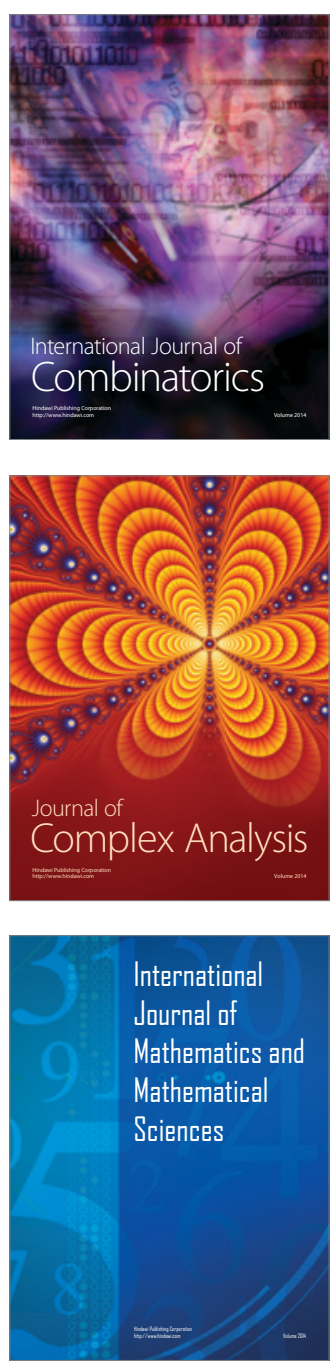
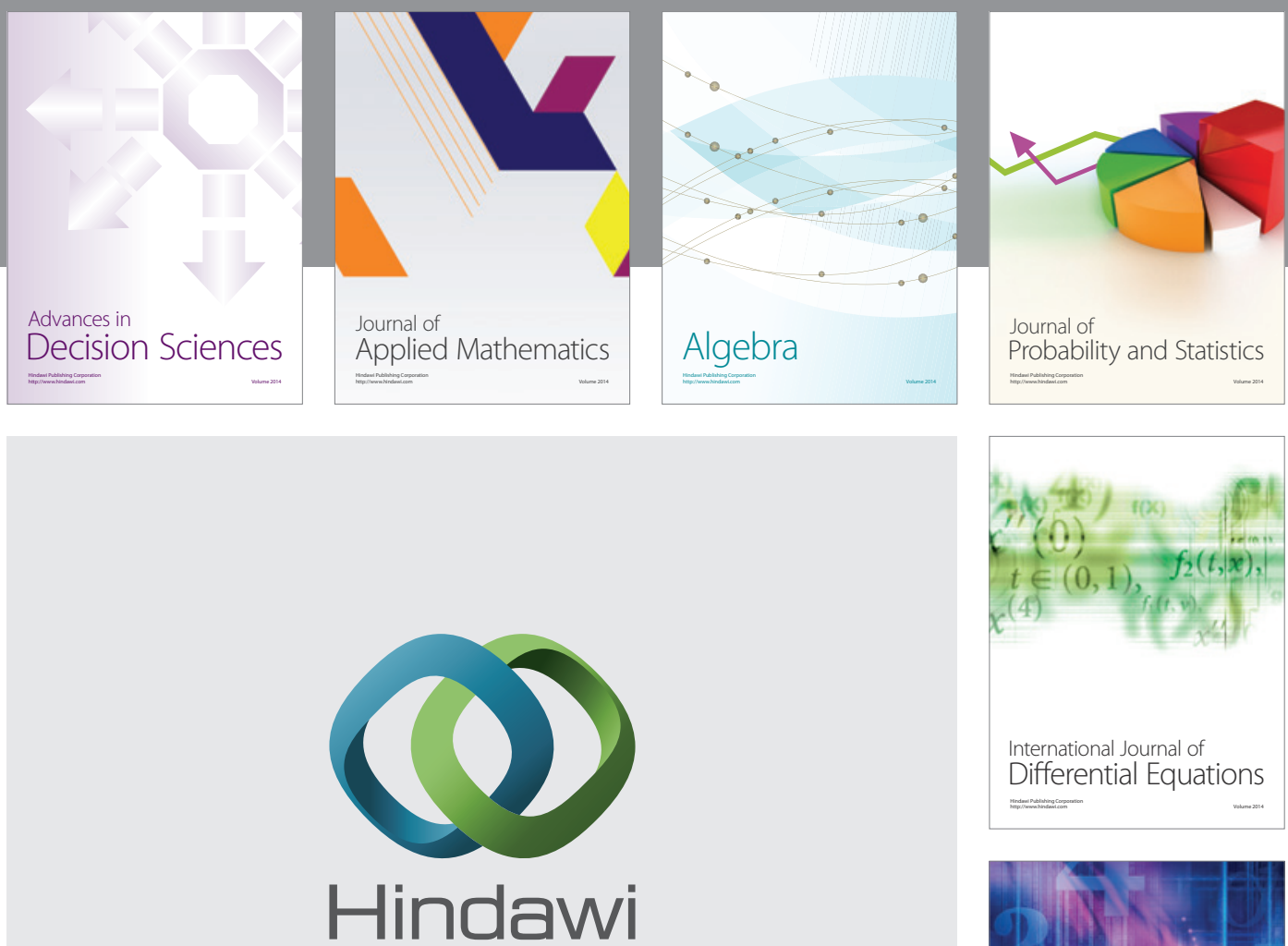

Submit your manuscripts at http://www.hindawi.com
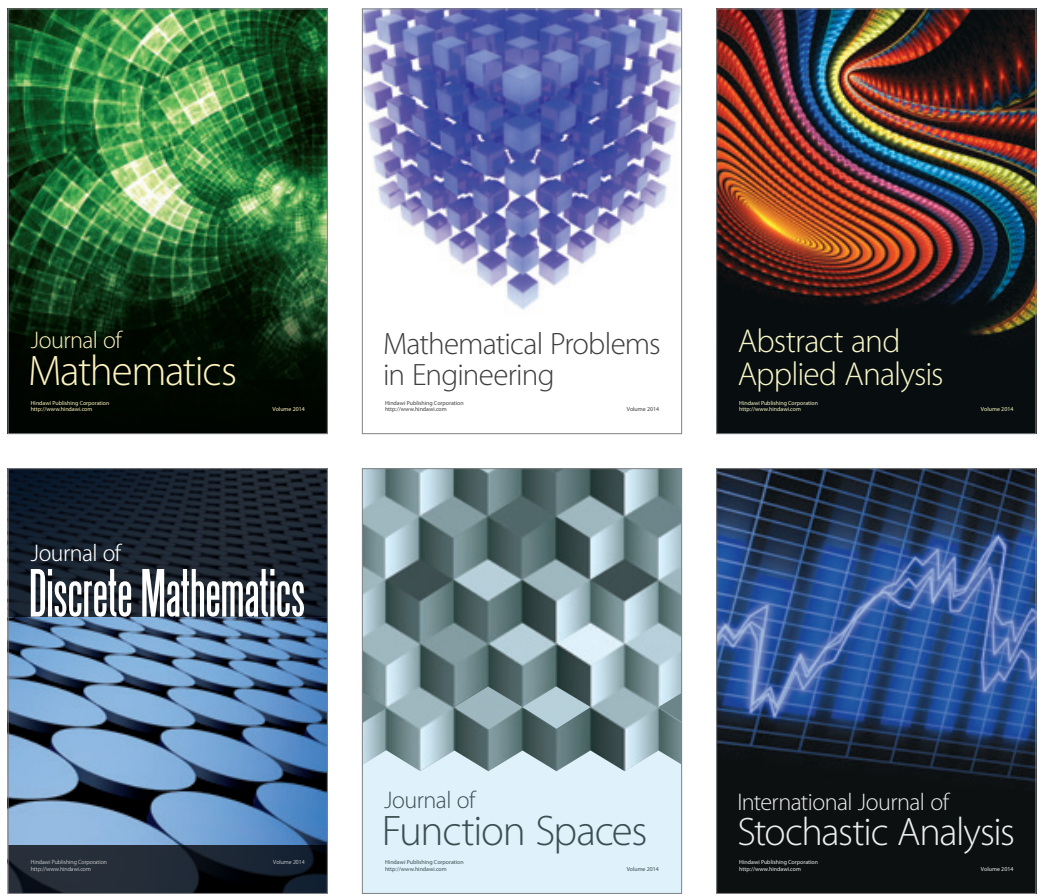

Journal of

Function Spaces

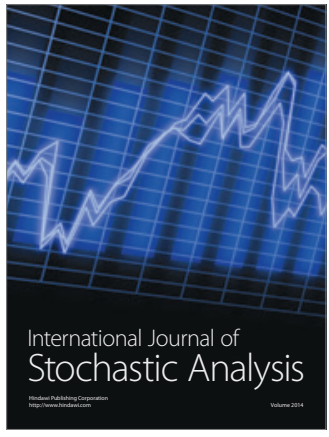

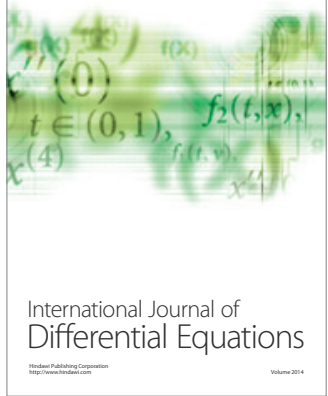
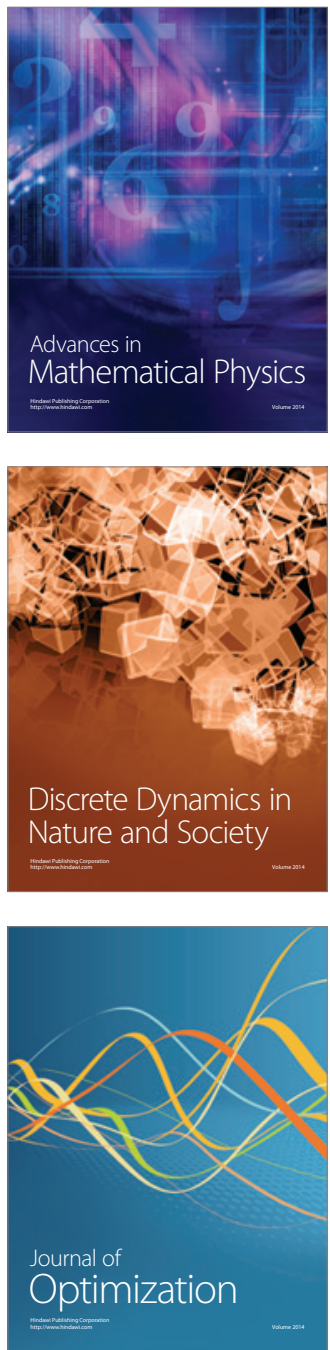